\title{
DEGRO practical guidelines for radiotherapy of breast cancer $\mathbf{V}$
}

\author{
Therapy for locally advanced and inflammatory breast cancer, as well as local therapy \\ in cases with synchronous distant metastases
}

\author{
Wilfried Budach $\cdot$ Christiane Matuschek $\cdot$ Edwin Bölke $\cdot$ Jürgen Dunst $\cdot$ Petra Feyer \\ Rainer FietkauHaase • Wulf Haase • Wolfgang Harms • Marc D. Piroth • \\ Marie-Luise Sautter-Bihl $\cdot$ Felix Sedlmayer $\cdot$ Rainer Souchon $\cdot$ Frederick Wenz $\cdot$ Rolf Sauer • \\ Breast Cancer Expert Panel of the German Society of Radiation Oncology (DEGRO)
}

Received: 27 March 2015 / Accepted: 9 April 2015 / Published online: 12 May 2015

(C) The Author(s) 2015. This article is published with open access at Springerlink.com

\begin{abstract}
Aim The purpose of this work is to give practical guidelines for radiotherapy of locally advanced, inflammatory and metastatic breast cancer at first presentation.

Methods A comprehensive survey of the literature using the search phrases "locally advanced breast cancer", "inflammatory breast cancer", "breast cancer and synchronous metastases", "de novo stage IV and breast cancer", and "metastatic breast cancer" and "at first presentation" restricted to "clinical trials", "randomized trials", "meta-analysis", "systematic review", and "guideline" was performed and supplemented by using references of the respective publica-
\end{abstract}

\footnotetext{
Prof. Dr. med. W. Budach $(\bowtie) \cdot$ C. Matuschek · E. Bölke

Klinik für Strahlentherapie und Radioonkologie,

University Hospital, Heinrich-Heine-University Düsseldorf,

Moorenstraße 5,

40225 Düsseldorf, Germany

e-mail: Wilfried.Budach@med.uni-duesseldorf.de

J. Dunst

University Hospital Schleswig-Holstein,

Luebeck, Germany

P. Feyer

Vivantes Hospital Neukoelln,

Berlin, Germany

R. Fietkau $\cdot$ R. Sauer

University Hospital Erlangen,

Erlangen, Germany

W. Haase

Formerly St.-Vincentius-Hospital,

Karlsruhe, Germany

W. Harms

St. Clara Hospital,

Basel, Switzerland
}

tions. Based on the German interdisciplinary S3 guidelines, updated in 2012, this publication addresses indications, sequence to other therapies, target volumes, dose, and fractionation of radiotherapy.

Results International and national guidelines are in agreement that locally advanced, at least if regarded primarily unresectable and inflammatory breast cancer should receive neoadjuvant systemic therapy first, followed by surgery and radiotherapy. If surgery is not amenable after systemic therapy, radiotherapy is the treatment of choice followed by surgery, if possible. Surgery and radiotherapy should be administered independent of response to neoadjuvant
M. D. Piroth

Helios Hospital,

Wuppertal, Germany

M.-L. Sautter-Bihl

Municipal Hospital,

Karlsruhe, Germany

F. Sedlmayer

Paracelsus Medical University Hospital,

Salzburg, Austria

R. Souchon

Formerly University Hospital Tübingen,

Tübingen, Germany

F. Wenz

Universitätsmedizin Mannheim,

Mannheim, Germany 
systemic treatment. In patients with a de novo diagnosis of breast cancer with synchronous distant metastases, surgery and radiotherapy result in considerably better locoregional tumor control. An improvement in survival has not been consistently proven, but may exist in subgroups of patients. Conclusion Radiotherapy is an important part in the treatment of locally advanced and inflammatory breast cancer that should be given to all patients regardless to the intensity and effect of neoadjuvant systemic treatment and the extent of surgery. Locoregional radiotherapy in patients with primarily distant metastatic disease should be prescribed on an individual basis.

Keywords Locally advanced breast cancer . Inflammatory breast cancer - Synchronous distant metastases $\cdot$ Radiotherapy $\cdot$ Guidelines

\section{DEGRO-Leitlinien für die Radiotherapie des Mammakarzinoms V}

Therapie des lokal fortgeschrittenen und inflammatorischen Mammakarzinoms sowie lokale Therapie bei Vorliegen synchroner Fernmetastasen

\section{Zusammenfassung}

Ziel Ziel der Arbeit ist die Entwicklung von Praxis-Leitlinien zur Strahlentherapie des lokal fortgeschrittenen einschließlich des inflammatorischen Mammakarzinoms sowie zur lokalen Therapie bei synchroner Fernmetastasierung.

Methoden Es erfolgte eine Literaturübersicht basierend auf den Suchbegriffen „locally advanced breast cancer", „,inflammatory breast cancer", „breast cancer and synchronous metastases“, „de novo stage IV and breast cancer“ und ,metastatic breast cancer" sowie ,at first presentation“. Stratifiziert wurde nach „clinical trials“, „randomized trials“, „meta-analysis“, „systematic review“ und „guideline“, ergänzt von kürzlich veröffentlichten Abstracts. Ergänzend zu den Stellungnahmen der deutschen interdisziplinären S3Leitlinie der Deutschen Krebsgesellschaft und der AWMF zum Mammakarzinom von 2012 bezieht diese Publikation Stellung zu den Indikationen, zur zeitlichen Abfolge mit anderen Therapien, zum Zielvolumen sowie zur Dosis und Fraktionierung der Strahlentherapie.

Ergebnisse Internationale und nationale Leitlinien stimmen darin überein, dass Patientinnen mit einem lokal fortgeschrittenen, zumindest primär als irresektabel eingestuften, sowie Patientinnen mit einem inflammatorischen Mammakarzinom zunächst eine neoadjuvante Systemtherapie erhalten sollen, gefolgt von einer Operation und einer Strahlentherapie. Falls nach der Systemtherapie eine Operation nicht durchführbar ist, sollte zunächst eine Radiotherapie erfolgen und anschließend noch einmal die Möglichkeit ei- ner Operation bedacht werden. Operation und Bestrahlung erfolgen unabhängig vom Ansprechen auf die neoadjuvante Systemtherapie. Bei Patientinnen mit primärer Fernmetastasierung führt die lokale Behandlung mittels Operation und/ oder Strahlentherapie zur besseren lokalen Tumorkontrolle. Ein Überlebensvorteil besteht wohl nicht, möglicherweise aber doch für einige Subgruppen der Patientinnen.

Schlussfolgerung Eine Strahlentherapie ist wichtiger Bestandteil der Behandlung lokal fortgeschrittener und inflammatorischer Mammakarzinome für alle betroffenen Patientinnen, unabhängig von der Intensität und dem Ergebnis der neoadjuvanten systemischen Therapie und des Ausmaßes einer eventuellen Chirurgie. Bei Patientinnen mit primärer Fernmetastasierung muss im Tumorboard die Indikation zur lokoregionären Strahlentherapie individuell gestellt werden.

Schlüsselwörter Lokal fortgeschrittenes Mammakarzinom - Inflammatorisches Mammakarzinom · Synchrone Fernmetastasierung · Strahlentherapie · Leitlinien

Tumors larger than $5 \mathrm{~cm}$ or tumors infiltrating the chest wall or skin (T3/T4), fixated axillary lymph nodes (N2), involved infra- or supraclavicular lymph nodes, or involved lymph nodes in the internal mammary chain (N3) are regarded as locally advanced breast cancer and are generally considered as not amenable for primary surgery. Inflammatory breast cancer (T4d) accounts for approximately $10-15 \%$ of locally advanced breast cancer. Historical results with radiotherapy as sole treatment reported 5-year survival rates in locally advanced breast cancer without systemic treatment of approximately $30 \%$ and in inflammatory breast cancer of $5 \%$ [1]. Distant metastases at the time of breast cancer diagnosis are detected in about $4 \%$ of all breast cancer patients, but are observed in $14 \%$ of T3 and $26 \%$ of T4 patients (Munich Cancer Registry 2013).

Nowadays, primary systemic treatment is considered standard of care for breast cancer patients with locally advanced and inflammatory breast cancer, as well as in patients with synchronous distant metastases [2,3]. Response to systemic treatment, especially pathologically complete response (pCR), has been shown to be associated with improved survival in all these situations [4]. The development of modern neoadjuvant treatment in less advanced breast cancer was based on experience in locally advanced and inflammatory breast cancer. Randomized clinical trials have focused on further development of more effective systemic treatment regimen, whereas radiotherapy was an integrated part of the treatment in these trials, since its importance for locoregional control was not challenged. This has changed to some extent, since a substantial portion of the patients are mean- 
while amenable for surgery after partial or complete clinical remission following neoadjuvant systemic treatment. Especially in case of pCR after mastectomy the impact of radiotherapy has been questioned.

The aim of this paper is to review the role of radiotherapy in locally advanced and inflammatory breast cancer, and for patients with synchronous distant metastases and to provide practical treatment guidelines relating to and supplementing the current German S3 guidelines and the previous practical DEGRO guideline [5].

\section{Locally advanced and inflammatory breast cancer}

\section{Infobox 1}

- RT-6 statement of the German S3 guideline (radiotherapy of locally advanced unresectable breast cancer) For patients with primarily inoperable or inflammatory breast cancer primary systemic therapy followed by surgery and postoperative radiotherapy is recommended (LOE 1b); recommendation grade A.

- If surgical resectability is not achieved after systemic treatment, radiotherapy - optionally also combined with concurrent systemic treatment-is indicated (GCP).

RT-4d statement of the German S3 guideline (radiotherapy after mastectomy)

- After neoadjuvant therapy, radiotherapy is indicated in accordance to the pretreatment $\mathrm{T}$ and $\mathrm{N}$ classification independent of the degree of response to the neoadjuvant treatment $(\mathrm{LoE} 2 \mathrm{a})$; recommendation grade $\mathrm{A}$.

Development of neoadjuvant chemotherapy

Although inflammatory breast cancer has a poorer outcome compared to other locally advanced breast cancers in prospective clinical trials and most retrospective evaluations treatment for both entities have mostly been identical. No more than sparse results of subgroup analyses were available from some reports. Therefore, differential treatment recommendations for locally advanced and inflammatory breast cancer cannot generally be given.

In 1997 the EORTC published the results of a phase III trial [6] on the treatment of locally advanced and inflammatory breast cancer. A total of 410 patients were randomized for either radiotherapy, radiotherapy plus chemotherapy (CMF), radiotherapy plus tamoxifen, or radiotherapy plus tamoxifen plus CMF. Surgery was not part of the planned treatment and was reserved for salvage treatment in case of local recurrence. Radiotherapy alone resulted in a 10-year overall survival of $13 \%$ and distant metastasis-free survival of $15 \%$ indicating some curative potential. Chemotherapy and hormonal treatment each produced a significant prolongation of the time to locoregional recurrence, time to distant metastases, and overall survival. Combined treatments provided the largest therapeutic effect. The 10 -year overall survival was improved by $8-15 \%$ by the addition of systemic treatments. A number of subsequent trials, designed to optimize systemic chemotherapy by introducing anthracyclines and taxanes, led to a stepwise improvement of 5-year survival in locally advanced cancer to about $70 \%$ and in inflammatory cancer to about $40 \%[7,8]$. In case of resectability after neoadjuvant chemotherapy, mastectomy followed by radiotherapy was performed in most patients. Importantly, subgroup analyses revealed that patients with a $\mathrm{pCR}$ in their mastectomy specimen had a substantially better overall survival than patients with partial response or stable disease $[4,9]$. This observation was the starting point for the use of pCR rates as a surrogate marker for overall survival in the further development of neoadjuvant treatment in breast cancer [10]. In these trials, incremental numbers of patients with moderately advanced breast cancers (e.g., cT2 disease) were included. The $\mathrm{pCR}$ rates in the recent German neoadjuvant chemotherapy breast cancer trials [11] were increased to an average of $21 \%$, showing considerable differences by the subtype of breast cancer. The pCR rates were on average lower in T3/T4 tumors and inflammatory cancer ranging between 13 and $16 \%$. In estrogen-receptor-negative cancers and undifferentiated cancers (G3), a substantially higher likelihood to achieve a pCR was observed as compared to estrogen-receptor-positive and well-differentiated cancers (odds ratios 3.8 and 5.8). The highest pCR rate of $41 \%$ was seen in patients with HER-2-positive tumors receiving 8-12 cycles of trastuzumab. The pCR rate achieved in inflammatory breast cancer is in the same range as for other locally advanced stages. This is probably a consequence of the higher likelihood of inflammatory cancer of being poorly differentiated, estrogen receptor negative, and HER-2 positive [11]. Using double HER-2-blocking therapies pCR rates in hormone-receptor-negative, undifferentiated tumors as high as $63 \%$ [12] have been reported. The results of the neoALTTO and ALTTO trials, however, shed some doubts whether higher $\mathrm{pCR}$ rates necessarily indicate better overall survival, since in spite of an observed doubling in the $\mathrm{pCR}$ by double HER-2 blockade in the neoALTTO trial [13], no benefit in overall survival was observed in the ALTTO trial testing the same regimen in the adjuvant setting [14]. Indeed a meta-analysis including almost 12,000 patients from 12 trials testing primary systemic therapy came to the same conclusion that albeit pCR is associated with a significantly improved overall survival, it is not a suitable surrogate parameter for overall or progression-free survival on a triallevel basis [15]. 
Locoregional treatment after neoadjuvant chemotherapy

Regardless of response to neoadjuvant treatment, the question arises to which extent additional local treatment further improves outcome. Results of randomized trials directly answering the question for the need of surgery and radiotherapy in this situation are not available. National and international guidelines [16-21] recommend radiotherapy for patients presenting with persistent unresectability after neoadjuvant chemotherapy, since radiotherapy has a curative potential in this situation [6]. Resectability should be assessed 6-12 weeks after completion of radiotherapy. Surgery is advised if a R0 resection seems achievable, although the benefit of surgery in this clinical setting has formally not been shown.

In stage III breast cancer patients with resectable disease after neoadjuvant chemotherapy, the effect of surgery and radiotherapy was investigated in three small randomized trials [22-24]. Patients were randomized after neoadjuvant chemotherapy (two trials with anthracyclines, one trial with CMF) to undergo either mastectomy or radiotherapy. Overall survival, disease-free survival, and locoregional tumor control were similar with both treatments. However, the locoregional recurrence rate at 2-5 years reached $40-60 \%$ with either treatment, indirectly indicating that patients should receive both surgery and radiotherapy. In none of these trials results according to response to the neoadjuvant treatment were reported. Thus, the question remains open whether surgery and radiotherapy are required in case of clinical complete response (cCR) or pCR after neoadjuvant chemotherapy.

Merajver et al. [4] treated 90 stage III breast cancer patients with nine cylces of an anthracycline-based neoadjuvant chemotherapy. After the last cycle of chemotherapy, a biopsy was taken from the initial tumor region. In case of pCR, patients received radiotherapy of the chest wall and regional lymph nodes without any surgery. Patients with residual tumor in the biopsy underwent mastectomy followed by the same radiotherapy. The omission of surgery in patients with pCR was not associated with a higher locoregional recurrence rate, which was approximately $20 \%$ at the 5 -year follow-up.

Ring et al. $(n=136,[25])$ and Daveau et al. $(n=165,[26])$ extracted from their prospective databases the outcome of stage III breast cancer patients with cCR after neoadjuvant chemotherapy who had received radiotherapy but no surgery and compared this with the outcome of patients with pCR after chemotherapy, who had received mastectomy and postoperative radiotherapy. The outcomes in terms of overall survival, disease-free survival, and distant metastases-free survival were almost identical. A trend towards better locoregional tumor control was observed for patients receiving surgery in addition to radiotherapy. In the absence of evidence from randomized trials, national and interna- tional guidelines [16-18, 27] recommend breast surgery after neoadjuvant systemic treatment regardless of the response to the neoadjuvant treatment if complete resection appears possible.

Whether mastectomy or breast-conserving surgery is preferable after neoadjuvant chemotherapy is another unanswered question. In a meta-analysis of randomized trials on neoadjuvant chemotherapy compared to adjuvant chemotherapy, overall survival and disease-free survival were identical. However, the rate of breast-conserving therapy was significantly higher in neoadjuvantly treated patients [28]. This increase in breast conservation rates was associated with a significantly higher rate of locoregional recurrence, which was, however, predominantly observed in three trials in which radiotherapy without surgery was more frequently used in the neoadjuvant arms of the trials. Although locally advanced primarily unresectable and inflammatory breast cancers were not treated in these trials, the result indirectly support the assumption that locally advanced and inflammatory breast cancers should, whenever possible, receive intensive locoregional treatment consisting of complete resection and locoregional radiotherapy.

Results of breast-conserving surgery after neoadjuvant chemotherapy in locally advanced and inflammatory breast cancer have been reported from a number retrospective evaluations [29]. Breast-conserving surgery in these cohorts was generally restricted to selected patients, whose tumors responded well to neoadjuvant treatment, were confined to one quadrant of the breast, and had no more signs of inflammatory disease. In this selected subgroup of patients after 5-10 years, in-breast recurrence rates between 2 and $23 \%$ have been reported [29], indicating that breast-conserving surgery is justifiable in appropriate patients.

The need of radiotherapy for patients with locally advanced breast cancer with residual disease after neoadjuvant treatment is not disputed in view of the convincing evidence from the EBCTCG meta-analysis [30] showing a significant survival advantage for postmastectomy radiotherapy in patients with $\mathrm{T} 4$ tumors or involved axillary lymph nodes. The need for radiotherapy in case of $\mathrm{pCR}$ after neoadjuvant chemotherapy has been challenged. The best available evidence to answer the question whether radiotherapy is necessary after mastectomy in case of pCR comes from two retrospective studies.

Huang et al. [31] assessed the clinical outcome of 676 breast cancer patients who had received six different neoadjuvant chemotherapy regimens within clinical trials followed by mastectomy and axillary dissection. Of these patients, 134 received no postmastectomy radiotherapy, whereas 542 underwent postmastectomy radiotherapy. As expected in a nonrandomized comparison, patients receiving radiotherapy had significantly more advanced $\mathrm{T}$ and $\mathrm{N}$ stages representing a bias in disadvantage of radiotherapy. In spite 
of this negative selection, locoregional tumor control and cause-specific survival were significantly better in irradiated patients with T4 tumors or more than four involved axillary lymph nodes associated with a trend towards an improved overall survival. Of 46 patients with locally advanced cancers, who had a pCR after neoadjuvant chemotherapy, 11 received radiotherapy. The actuarial locoregional recurrence rate at 10 and 14 years was $3 \%$ in irradiated patients compared to $33 \%$ without radiotherapy $(p=0.006)$.

McGuire et al. [32] identified 106 patients with stage IIIII breast cancer (69\% stage III, no inflammatory cancers) treated with neoadjuvant chemotherapy ( $92 \%$ anthracycline-based chemotherapy, $38 \%$ with taxane) mastectomy and axillary lymph node dissection, who had a pCR. Of these, 34 patients underwent postmastectomy radiotherapy and 72 no radiotherapy. Irradiated patients had significantly larger tumors than unirradiated patients. Regardless of this unfavorable bias, patients receiving postmastectomy radiotherapy experienced significantly better locoregional tumor control (absolute $\sim+20 \%$ ), distant metastases-free survival (absolute $\sim+37 \%$ ), and overall survival (absolute $\sim+40 \%$ ) 5 year after treatment. Both studies, although not randomized, led consistently to the recommendation in national and international guidelines $[16-19,33]$ to use radiotherapy in patients with a pCR after neoadjuvant chemotherapy. Whether a comparable benefit can also be expected for patients with HER-2-positive breast cancer receiving antiHER-2 therapies is unknown. In absence of reliable evidence regarding this question, radiotherapy is also advised in these patients in the guidelines.

Attempts to identify subgroups of patients with locally advanced breast cancer based on molecular profiling, like in early breast cancer, have not been successful so far [34]. Although it has been shown that luminal A and B breast cancers have lower propensity for locoregional recurrences than HER-2-positive and triple-negative breast cancers [35], radiotherapy decreases the locoregional recurrence rate independent of the molecular subtype approximately by the factor 3-4 [36]. However, there is some evidence that the benefit of radiotherapy in terms of locoregional control translates into a smaller survival benefit in HER-2 positive and triple negative compared to hormone-receptor-positive breast cancer $[36,37]$. Currently, differential indications for radiotherapy based on molecular profiles are not recommended in national or international guidelines outside from clinical trials.

\section{Comments and conclusion of the DEGRO panel}

- The DEGRO panel recommends locoregional radiotherapy as part of the treatment for all patients with locally advanced and inflammatory breast cancer regardless of the response to neoadjuvant systemic treatment. This is in accordance with the German S3 guideline.
Timing of radiotherapy

Radiotherapy in locally advanced or inflammatory breast cancer was administered after neoadjuvant chemotherapy and mastectomy in most clinical investigations. In case of remaining unresectability after completion of chemotherapy, radiotherapy is given followed by surgical resection, if clinically possible. Interestingly, the optimal timing of radiotherapy in the management of locally advanced breast cancer and IBC has not been systematically investigated. In a number of phase II trials, sequential radiotherapy after neoadjuvant chemotherapy as well as concurrent chemoradiation were analyzed in stage II and III breast cancer [38]. Overall survival, disease-free survival, and locoregional control in these trials were not substantially different from published data for postoperative radiotherapy. The pCR rates in these trials vary between $16 \%$ [39] and $45 \%$ [40]. On average, trials using concurrent chemoradiation reported higher pCR rates than trials on sequential chemoradiation. In none of these trials were HER-2 antagonists used for HER-2-positive cancer. Unfortunately, subgroup analyses differentiating $\mathrm{pCR}$ rates by molecular subtypes are not available from most of these trials. The available data indicate that, similar to the observations for neoadjuvant chemotherapy, pCR rates after additional radiotherapy are higher in hormone-receptor-negative disease than in hormone-receptor-positive breast cancer. However, the reported pCR rates of $18 \%(n=57)$ in the data of Adams et al. [41] and 29\% $(n=241)$ in the data of Matuschek et al. [42] for hormone-receptor-positive breast cancer compare favorably to the experience in the German neo-adjuvant chemotherapy trials, in which a pCR of $13 \%(n=2150)$ was reported for hormone-receptor-positive disease. Interestingly enough, pCR rates increased with incremental intervals from completion of radiotherapy to surgery in the data of Matuschek et al. [42], indicating that cancer cell death after radiotherapy takes several months for completion and occurs typically postmitotic in breast cancer.

The reported acute and late toxicities in the trials on sequential or concurrent chemoradiation in breast cancer were mostly grade I and II. In early breast cancer, sequential and concurrent adjuvant chemoradiation were tested in a number of randomized controlled trials in which breast-conserving surgery was generally performed. In a Cochrane review of these trials [43], concurrent chemoradiation resulted in significantly more skin reactions (odds ratio $1.46,95 \% \mathrm{CL} 1.00-2.14$ ), esophagitis (odds ratio $1.44,95 \%$ CL 1.03-2.02), and hematotoxicity (odds ratio $1.43,95 \%$ CL 1.01-2.03) compared to sequential treatment, whereas late toxicity was comparable. No difference was seen in overall survival and locoregional recurrence rates. Whether these results can be transferred to the neoadjuvant 
setting in locally advanced breast cancer is unknown in the absence of randomized trials.

Since in locally advanced and inflammatory breast cancer mastectomy is considered as the standard surgical procedure, many patients, especially those of younger age, wish immediate or sequential breast reconstruction. However, the optimal sequencing of breast reconstruction and radiotherapy is another unanswered question. Three options have been investigated:

- mastectomy followed by radiotherapy followed by reconstructive surgery;

- mastectomy with immediate reconstructive surgery followed by radiotherapy;

- radiotherapy followed by mastectomy with immediate reconstructive surgery.

In the absence of randomized trials, all comparisons remain inconclusive. From the available data for the different strategies, the oncological outcome is presumably similar [44]. There seems to be a tendency that early complications are more frequently observed in patients having radiotherapy first. Late complications occur more often in patients undergoing breast reconstruction first [45]. If an immediate autologous reconstruction is planned, radiotherapy before mastectomy and immediate reconstruction is theoretically advantageous, because the autologous tissue used for reconstruction does not receive radiotherapy at any time and consequently will not develop radiation-induced fibrosis. This approach was tested in a prospective multi-institutional investigation in Düsseldorf: Gerlach et al. [46] reported the early results of a preoperative chemoradiation in 132 breast cancer patients ( $90 \%$ stage II and III) of whom 82 received transverse rectus abdominus myocutaneous (TRAM) or latissimus dorsi flab reconstruction at a median time of 8 weeks after completion of radiotherapy. In a nonrandomized comparison with 62 patients receiving neoadjuvant chemotherapy without radiotherapy before breast surgery, they did not find a higher rate of surgical complications. Roth et al. [47] confirmed these results in the long-term follow-up on the complete cohort of 315 patients and reported a favorable long-term oncological outcome compared to a similar group of patients treated with postoperative radiotherapy.

\section{Comments and conclusions of the DEGRO panel}

- The optimal timing of radiotherapy is not well investigated. Most data are available for conducting radiotherapy after neoadjuvant systemic treatment and mastectomy, or in case of persistent unresectability after completion of neoadjuvant treatment.

- If immediate autologous reconstruction is intended, radiotherapy and concurrent chemoradiation before surgery are alternative options.
Target volumes, dose, and fractionation of radiotherapy

The vast majority of patients with locally advanced and inflammatory breast cancer in clinical trials received chest wall radiotherapy and radiotherapy to the regional lymph nodes. In the German neoadjuvant trials [11], radiotherapy was administered according to the current German S3 guidelines at that time. Although no information on the actually administered radiotherapy has been published from any of these trials, most likely radiation oncologist treated patients in adherence to the guidelines, permitting the conclusion that all patients with locally advanced and inflammatory breast cancer received radiotherapy of the chest wall and the majority radiotherapy of the supra- and infraclavicular lymph nodes. Most likely, only a minority of the patients has received radiotherapy to the internal mammary lymph nodes in these trials. Recently, new data emerged, indicating that radiotherapy of the internal mammary lymph nodes results in a small, but statistically significant survival benefit [48-50]. This benefit was more pronounced in patients receiving both chemotherapy and endocrine therapy [49], but which was less substantial in N3 disease. Radiotherapy of the internal mammary lymph nodes (IMC) was not significantly associated with increased cardiotoxicity after a median follow-up of 10.9 years. However, trastuzumab or other anti-HER2 agents were not given in this trial.

Based on this new data, the DEGRO panel has already published recommendation for the treatment of the regional lymph nodes in breast cancer [51]. The DEGRO panel recommends radiotherapy of the chest wall or breast and the supra- and infraclavicular lymph nodes in all patients with locally advanced and inflammatory breast cancer regardless of the response to systemic therapy. Radiotherapy of the internal mammary lymph nodes should be considered in patients, who do not receive anti-HER2 treatments.

If a surgical resection after neoadjuvant treatment is not possible or declined by the patient, most investigators defined the macroscopic tumor with some safety margin (exact description mostly not published) as clinical target volume for an additional boost dose.

In the majority of clinical trials on radiotherapy after mastectomy in locally advanced breast cancer, conventionally fractionated radiotherapy with $1.8-2.0$ Gy in 5 fractions per week to total doses of 45.0-50.4 Gy was used. Hypofractionated radiotherapy with 2.3-3.7 Gy per fraction in 2-5 fractions per week to total doses of 30-50 Gy were applied preferentially in the United Kingdom, Scandinavia, and Canada [52]. Since from today's point of view the total dose in some of these hypofractionated regimens was not adequately reduced in relation to the increase in dose per fraction, long-term side effects, especially plexopathia, were frequently reported [53]. 
Modern hypofractioned regimens that were tested predominantly in early breast cancer, like the START A (3.2 Gy per fraction, $2-3$ times per week to $41.6 \mathrm{~Gy}$ ) and the START B scheme (2.66 Gy per fraction, 5 times per week to $40 \mathrm{~Gy}$ ) implemented an adequate reduction of the total dose [54]. As a consequence these schedules should not be associated with a higher probability of severe late effects than conventionally fractionated regimens.

Indeed, at a median follow-up of almost 10 years cosmetic results with hypofractionation were not impaired, and in the few patients receiving supra- and infraclavicular lymph node irradiation, only in 1 out of 211 patients (95\% CL 0$2.6 \%$ ) in the START A trial, and in none out of 82 patients (95\% CL 0-4.4\%) in the START B trial was plexopathia reported [54]. Since plexopathia may occur as late as 25 years after radiotherapy [55], its final incidence is expected to be higher. None of the patients in the randomized trials on hypofractionation compared to conventional fractionated radiotherapy in early breast cancer received radiotherapy of the internal mammary lymph nodes. Radiation-induced heart toxicity is particularly associated with radiotherapy of the internal mammary lymph nodes [56]. Since one cannot exclude that hypofractionated radiotherapy to the internal mammary lymph node may increase cardiac toxicity and hypofractionated radiotherapy to the supra- and infraclavicular lymph nodes increase late incidence of plexopathia, the DEGRO panel recommends conventionally fractionated radiotherapy with 1.8-2.0 Gy per fraction 5 times per week to total doses of 45-50.4 Gy for the therapy of the regional lymph nodes in the adjuvant situation. Because chest wall radiotherapy is almost always indicated at the same time, the same fractionation regimen should be used for radiotherapy of the chest wall. If radiotherapy is administered without previous surgery the same arguments regarding the dose and fractionation apply as regional nodal irradiation is generally indicated. An additional sequential boost dose of 10-20 Gy to the macroscopic tumor volume is recommended in this situation as conventionally fractionated percutaneous radiotherapy or as brachytherapy. Alternatively, the boost dose may be given as simultaneous integrated boost using 2.22.4 Gy per fraction. A boost dose to remaining macroscopic lymph node metastases maybe considered, if the dose to the brachial plexus is kept below a 2 Gy equivalent dose $(\alpha / \beta=3$ Gy) of about 54 Gy. Radiotherapy should be delivered in supine position using a dedicated breast board.

\section{Comments and conclusions of the DEGRO panel}

- The clinical target volume (CTV) at the chest wall should include the intercostal spaces in the region of the (resected) breast.

- The CTVs for the radiotherapy of the regional lymph nodes should be based on published international consensus guidelines [57]; however, adaptations may be necessary in locally advanced and inflammatory disease to ensure that any macroscopic disease is included into the CTV with adequate safety margins.

- 3D-CRT or IMRT treatment planning is recommended using algorithms providing a reliable dose prediction in the lungs, lung chest wall, and lung-heart interface (e.g., Monte Carlo or Collapsed cone). If IMRT is used, which may be especially advantageous if the internal mammary lymph nodes need to be irradiated (Fig. 1a, b, c), low dose exposure to large parts of the lungs and contralateral breast should be kept as small as possible.

Locoregional radiotherapy in patients with synchronous distant metastases

Radiotherapy to the breast, axillary, infra, and supraclavicular lymph nodes in metastatic breast cancer is frequently performed to obviate or relieve symptoms, but is not thought to have an impact on survival [58]. However, according to the results of a number retrospective studies on breast cancer [59-66], local treatment of the primary tumor by radiotherapy or surgery or both may improve overall survival. The data on surgical treatment of the primary site were comprehensively reviewed by Ruiterkamp et al. [67], whereas the present paper focuses on the effect of radiotherapy with/or without surgery.

Le Scodan et al. [66] retrospectively analyzed the outcome of 581 patients with metastatic breast cancer of whom
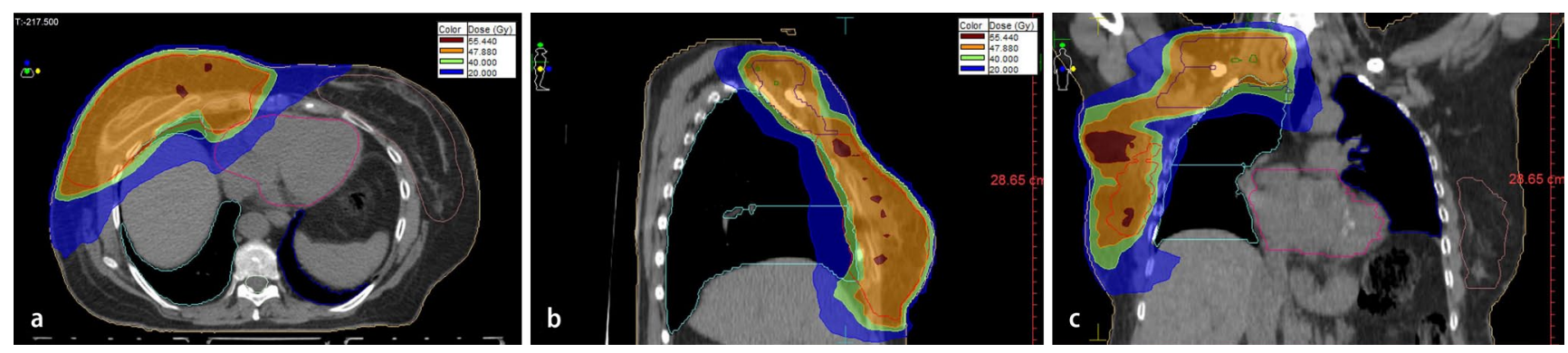

Fig. 1 Treatment plan irradiating the right chestwall (including a tissue expander) and the right supraclavicular and mammary interna lymph node regions. a axial view. b sagittal view. c coronal view 
320 received locoregional treatment after detection of distant metastases. Locoregional therapy comprised radiotherapy in 249 patients (78\%), surgery of the primary tumor with adjuvant irradiation in 41 patients $(13 \%)$, and surgery alone in 30 patients (9\%). Median follow-up was 39 months. A 3-year overall survival rate of $43.4 \%$ was observed in patients receiving locoregional treatment of the primary, but only $26.7 \%$ in patients receiving no locoregional therapy $(p<00002)$. The advantage in overall survival in favor of locoregional treatment was notably marked in patients with visceral metastases, whereas no survival benefit was seen in patients with bone metastases. Treatment of the primary tumor was an independent prognostic factor in multivariate analysis (hazard ratio: $0.70 ; 95 \%$ CI: $0.58-0.85 ; p<0.0002$ ). Radiotherapy of the breast or chest wall and the infra- and supraclavicular lymph nodes was used in most patients. A mean total dose of 48.7 Gy was given within 4-5 weeks to the majority of patients. Most patients, who did not receive breast surgery $(n=249)$, obtained an extra boost irradiation to the primary tumor site with an average dose of $23 \mathrm{~Gy}$. A trend towards longer survival was observed for patients receiving both surgery and radiotherapy.

A group of investigators from India reported the preliminary results of a randomized controlled trial [68]. Patients with synchronous metastatic breast cancer were treated with anthracycline-based chemotherapy with or without taxane. Patients with complete or partial response to chemotherapy $(n=350)$ were randomized to undergo locoregional treatment or not. Patients allocated to locoregional treatment received breast conservation surgery or mastectomy plus axillary lymph node dissection followed by radiotherapy. Patients randomized to the control arm were followed up without surgery or radiotherapy. Both groups received standard endocrine therapy after completion of chemotherapy, if indicated. Stratification by endocrine receptor status, site of metastases (visceral vs. bone vs. both), and number of metastatic lesions ( $\leq 3$ vs. $>3$ ) resulted in a well-balanced distribution of these factors in the treatment arms. At a median follow-up of 17 months, the median survival time was almost identical in both arms (18.8 and 20.5 months, $\mathrm{HR}=1.07,95 \% \mathrm{CI}=0.82-1.40, p=0.60)$. After adjustment for known prognostic factors using the Cox model, still no survival benefit could be detected for the additional use of locoregional treatment. Actuarial locoregional progression at 2 years as first event was observed in $11 \%$ of patients with locoregional treatment and in $52 \%$ without locoregional treatment $(p<0.001)$, whereas distant progression at 2 years as first event was observed in $72 \%$ of patients with locoregional treatment and in $52 \%$ without locoregional treatment $(p=0.01)$. The latter observation should not be misinterpreted in a manner that local therapy results in a higher rate of distant metastases, but that the increased rate of distant metastases as first event is simply a consequence of the better locoregional tumor control in the arm with locoregional treatment. Subgroup analyses did not indicate different results depending on hormone receptor status, HER-2 expression status, site of metastases, and number of metastatic lesions.

The second randomized trial was conducted by a group from Turkey [69]. Patients with metastatic breast cancer at the time of diagnosis were randomized to undergo locoregional treatment $(n=140)$ consisting of breast-conserving surgery followed by radiotherapy or mastectomy without radiotherapy or to receive no locoregional treatment $(n=138)$. The groups were comparable regarding age, BMI, hormone receptor and HER-2 status, tumor type, and size. Median follow-up was 21 months. After 54 months overall survival was $35 \%$ with locoregional treatment and $31 \%$ without locoregional treatment $(p=0.24)$. In the subgroups of hormone-receptor-positive disease, young patients $(<50$ years), and patients with a solitary bone metastasis, overall survival was significantly improved. Patients with triplenegative disease had significantly worse survival in the arm with locoregional treatment. The partially conflicting results of the randomized trials that have not yet been fully published, which in synopsis with the retrospective investigation do not allow for a final conclusion. Overall, the benefit is probably limited; however, subgroups may have an advantage.

Different mechanisms have been discussed about how locoregional treatment of the primary site could prolong survival. Uncontrolled locoregional disease can induce edema, infection, and thromboses and result in life-threatening problems. The persistent primary tumor could be a source of continuous seeding of further distant disease. Recent experimental data indicate that radiation-induced tumor cell necrosis can result in an improved dendritic-cell-mediated antitumoral immune response [70].

\section{Comments and conclusions of the DEGRO panel}

- Based on the available data, no general recommendations can be given for locoregional treatment outside of clinical trials. The decision of who is a candidate for locoregional radiotherapy should be made in an interdisciplinary board. The optimal dose and fractionation of radiotherapy in this situation has not been investigated.

- In patients with longer life expectancy, the same radiotherapy regimens as recommended for locally advanced and inflammatory breast cancer should be used.

\section{Compliance with ethical guidelines}

Conflict of interest The corresponding author states that there are no conflicts of interest.

The accompanying manuscript does not include studies on humans or animals. 
Open Access This article is distributed under the terms of the Creative Commons Attribution License which permits any use, distribution, and reproduction in any medium, provided the original author(s) and source are credited.

\section{References}

1. Buzdar AU, Hortobagyi GN, Asmar L, Theriault RL, Rahman Z, McNeese $M$ et al (1997) Prospective randomized trial of paclitaxel alone versus 5-fluorouracil/doxorubicin/cyclophosphamide as induction therapy in patients with operable breast cancer. Semin Oncol 24:S17-31-S17-34

2. Pohl F (2014) Significant cardiotoxicity of trastuzumab in adjuvant systemic therapy of elderly patients with breast cancer. Strahlenther Onkol 190:699-700

3. Corradini S, Niemoeller OM, Niyazi M, Manapov F, Haerting M, Harbeck $\mathrm{N}$ et al (2014) Timing of radiotherapy following breastconserving surgery: outcome of 1393 patients at a single institution. Strahlenther Onkol 190:352-357

4. Merajver SD, Weber BL, Cody R, Zhang D, Strawderman M, Calzone KA et al (1997) Breast conservation and prolonged chemotherapy for locally advanced breast cancer: the University of Michigan experience. J Clin Oncol 15:2873-2881

5. Sautter-Bihl ML, Souchon R, Budach W, Sedlmayer F, Feyer P, Harms W et al (2008) DEGRO practical guidelines for radiotherapy of breast cancer II. Postmastectomy radiotherapy, irradiation of regional lymphatics, and treatment of locally advanced disease. Strahlenther Onkol 184:347-353

6. Bartelink H, Rubens RD, van der Schueren E, Sylvester R (1997) Hormonal therapy prolongs survival in irradiated locally advanced breast cancer: a European Organization for Research and Treatment of Cancer Randomized Phase III Trial. J Clin Oncol $15: 207-215$

7. Rehman S, Reddy CA, Tendulkar RD (2012) Modern outcomes of inflammatory breast cancer. Int J Radiat Oncol Biol Phys $84: 619-624$

8. Scotti V, Desideri I, Meattini I, Di Cataldo V, Cecchini S, Petrucci A et al (2013) Management of inflammatory breast cancer: focus on radiotherapy with an evidence-based approach. Cancer Treat Rev 39:119-124

9. Palangie T, Mosseri V, Mihura J, Campana F, Beuzeboc P, Dorval T et al (1994) Prognostic factors in inflammatory breast cancer and therapeutic implications. Eur J Cancer 30A:921-927

10. Untch M, Konecny GE, Paepke S, von Minckwitz G (2014) Current and future role of neoadjuvant therapy for breast cancer. Breast 23:526-537

11. von Minckwitz GU, Kummel S et al (2011) Impact of treatment characteristics on response of different breast cancer phenotypes: pooled analysis of the German neo-adjuvant chemotherapy trials. Breast Cancer Res Treat 125:145-156

12. Gianni L, Pienkowski T, Im YH, Roman L, Tseng LM, Liu MC et al (2012) Efficacy and safety of neoadjuvant pertuzumab and trastuzumab in women with locally advanced, inflammatory, or early HER2-positive breast cancer (NeoSphere): a randomised multicentre, open-label, phase 2 trial. Lancet Oncol 13:25-32

13. Azim HA Jr, Agbor-Tarh D, Bradbury I, Dinh P, Baselga J, Di Cosimo $\mathrm{S}$ et al (2013) Pattern of rash, diarrhea, and hepatic toxicities secondary to lapatinib and their association with age and response to neoadjuvant therapy: analysis from the NeoALTTO trial. J Clin Oncol 31:4504-4511
14. Piccart-Gebhart MJ (2014) First results from the phase III ALTTO trial (BIG 2-06; NCCTG [Alliance] N063D) comparing one year of anti-HER2 therapy with lapatinib alone (L), trastuzumab alone $(T)$, their sequence $(T \rightarrow L)$, or their combination $(T+L)$ in the adjuvant treatment of HER2-positive early breast cancer (EBC). J Clin Oncol 32:5s

15. Cortazar P, Zhang L, Untch M, Mehta K, Costantino JP, Wolmark $\mathrm{N}$ et al (2014) Pathological complete response and long-term clinical benefit in breast cancer: the $\mathrm{CTNeoBC}$ pooled analysis. Lancet 384:164-172

16. AWMF (2012) Interdisziplinäre S3-Leitlinie für die Diagnostik, Therapie und Nachsorge des Mammakarzinoms. Leitlinienprogramm Onkol

17. (AGO) (2014) AGO diagnosis and treatment of patients with primary and metastatic breast cancer

18. National Comprehensive Cancer Network V (2013) NCCN clinical practice guidelines in oncology: breast cancer. (Version 1.2013.)

19. BCDS G (2011) Breast Cancer Evidence-based Series (EBS) and Practical Guidelines (PG). Cancer Care Ontario

20. Council NHaMR (2001) Clinical practice guidelines for the management of advanced breast cancer

21. (SIGN) (2013) SIGN Treatment of primary breast cancer. A national clinical guideline

22. Perloff M, Lesnick GJ, Korzun A, Chu F, Holland JF, Thirlwell MP et al (1988) Combination chemotherapy with mastectomy or radiotherapy for stage III breast carcinoma: a Cancer and Leukemia Group B study. J Clin Oncol 6:261-269

23. Mourali N, Tabbane F, Muenz LR, Behi J, Ben Moussa F, Jaziri M et al (1993) Ten-year results utilizing chemotherapy as primary treatment in nonmetastatic, rapidly progressing breast cancer. Cancer Invest 11:363-370

24. De Lena MV, Valagussa P et al (1981) Multimodal treatment for locally advanced breast cancer. Result of chemotherapy-radiotherapy versus chemotherapy-surgery. Cancer Clin Trials 4:229-236

25. Ring A, Webb A, Ashley S, Allum WH, Ebbs S, Gui G et al (2003) Is surgery necessary after complete clinical remission following neoadjuvant chemotherapy for early breast cancer? J Clin Oncol 21:4540-4545

26. Daveau C, Savignoni A, Abrous-Anane S, Pierga JY, Reyal F, Gautier C et al (2011) Is radiotherapy an option for early breast cancers with complete clinical response after neoadjuvant chemotherapy? Int J Radiat Oncol Biol Phys 79:1452-1459

27. Network SIG 84 Management of breast cancer in women. A national clinical guideline

28. Mauri D, Pavlidis N, Ioannidis JP (2005) Neoadjuvant versus adjuvant systemic treatment in breast cancer: a meta-analysis. J Natl Cancer Inst 97:188-194

29. Alm El-DinMA, Taghian AG (2009) Breast conservation therapy for patients with locally advanced breast cancer. Semin Radiat Oncol 19:229-235

30. McGale P, Taylor C, Correa C, Cutter D, Duane F, Ewertz M et al (2014) Effect of radiotherapy after mastectomy and axillary surgery on 10-year recurrence and 20-year breast cancer mortality: meta-analysis of individual patient data for 8135 women in 22 randomised trials. Lancet 383:2127-2135

31. Huang EH, Tucker SL, Strom EA, McNeese MD, Kuerer HM, Buzdar AU et al (2004) Postmastectomy radiation improves localregional control and survival for selected patients with locally advanced breast cancer treated with neoadjuvant chemotherapy and mastectomy. J Clin Oncol 22:4691-4699

32. McGuire SE, Gonzalez-Angulo AM, Huang EH, Tucker SL, Kau SW, Yu TK et al (2007) Postmastectomy radiation improves the outcome of patients with locally advanced breast cancer who achieve a pathologic complete response to neoadjuvant chemotherapy. Int J Radiat Oncol Biol Phys 68:1004-1009 
33. Network SIG 84 Management of breast cancer in women-A national clinical guideline. 2005

34. Kreike B, Halfwerk H, Armstrong N, Bult P, Foekens JA, Veltkamp SC et al (2009) Local recurrence after breast-conserving therapy in relation to gene expression patterns in a large series of patients. Clin Cancer Res 15:4181-4190

35. Nguyen PL, Taghian AG, Katz MS, Niemierko A, Abi Raad RF, Boon WL et al (2008) Breast cancer subtype approximated by estrogen receptor, progesterone receptor, and HER-2 is associated with local and distant recurrence after breast-conserving therapy. $\mathrm{J}$ Clin Oncol 26:2373-2378

36. Kyndi M, Sorensen FB, Knudsen H, Overgaard M, Nielsen HM, Overgaard J et al (2008) Estrogen receptor, progesterone receptor, HER-2, and response to postmastectomy radiotherapy in high-risk breast cancer: the Danish Breast Cancer Cooperative Group. J Clin Oncol 26:1419-1426

37. Darby S, McGale P, Correa C, Taylor C, Arriagada R, Clarke M et al (2011) Effect of radiotherapy after breast-conserving surgery on 10 -year recurrence and 15 -year breast cancer death: meta-analysis of individual patient data for 10,801 women in 17 randomised trials. Lancet 378:1707-1716

38. Kaidar-Person O, Kuten A, Belkacemi Y (2014) On behalf of A. Primary systemic therapy and whole breast irradiation for locally advanced breast cancer: a systematic review. Crit Rev Oncol Hematol 92:143-152

39. Formenti SC, Truong MT, Goldberg JD, Mukhi V, Rosenstein B, Roses D et al (2004) Prone accelerated partial breast irradiation after breast-conserving surgery: preliminary clinical results and dose-volume histogram analysis. Int J Radiat Oncol Biol Phys 60:493-504

40. Shanta V, Swaminathan R, Rama R, Radhika R (2008) Retrospective analysis of locally advanced noninflammatory breast cancer from Chennai, South India, 1990-1999. Int J Radiat Oncol Biol Phys 70:51-58

41. Adams S, Chakravarthy AB, Donach M, Spicer D, Lymberis S, Singh B et al (2010) Preoperative concurrent paclitaxel-radiation in locally advanced breast cancer: pathologic response correlates with five-year overall survival. Breast Cancer Res Treat 124:723-732

42. Matuschek C, Bolke E, Roth SL, Orth K, Lang I, Bojar H et al (2012) Long-term outcome after neoadjuvant radiochemotherapy in locally advanced noninflammatory breast cancer and predictive factors for a pathologic complete remission: results of a multivariate analysis. Strahlenther Onkol 188:777-781

43. James ML, Lehman M, Hider PN, Jeffery M, Francis DP, Hickey BE (2008) Fraction size in radiation treatment for breast conservation in early breast cancer. Cochrane Database Syst Rev 3:CD003860

44. D'Souza N, Darmanin G, Fedorowicz Z (2011) Immediate versus delayed reconstruction following surgery for breast cancer. Cochrane Database Syst Rev 7:CD008674 (Online)

45. Adesiyun TA, Lee BT, Yueh JH, Chen C, Colakoglu S, Anderson $\mathrm{KE}$ et al (2011) Impact of sequencing of postmastectomy radiotherapy and breast reconstruction on timing and rate of complications and patient satisfaction. Int J Radiat Oncol Biol Phys 80:392-397

46. Gerlach B, Audretsch W, Gogolin F, Konigshausen T, Rohn R, Schmitt $G$ et al (2003) Remission rates in breast cancer treated with preoperative chemotherapy and radiotherapy. Strahlenther Onkol 179:306-311

47. Roth SL, Audretsch W, Bojar H, Lang I, Willers R, Budach W (2010) Retrospective study of neoadjuvant versus adjuvant radiochemotherapy in locally advanced noninflammatory breast cancer: survival advantage in cT2 category by neoadjuvant radiochemotherapy. Strahlenther Onkol 186:299-306
48. Whelan IO TJ, Ackerman I, Chapman JW, Chua B, Nabid A, Vallis KA, White JR, Rousseau P, Fortin A, Pierce LJ, Manchul L, Craighead P, Nolan MC, Bowen J, McCready DR, Pritchard KI, Levine MN, Parulekar W (2011) NCIC-CTG MA.20: An intergroup trial of regional nodal irradiation in early breast cancer. J Clin Oncol 29s(LBA1003)

49. Philip Poortmans HS, Carine K, Volker B, Philippe M, Maria CV, Sandra C, Alain F, Harry B, Walter VB (2013) Irradiation of the internal mammary and medial supraclavicular lymph nodes in stage I to III breast cancer: 10 years results of the EORTC Radiation Oncology and Breast Cancer Groups phase III trial 22922/10925. Radiother Oncol Supp 47 (Suppl 2). (EJC)

50. Budach W, Kammers K, Boelke E, Matuschek C (2013) Adjuvant radiotherapy of regional lymph nodes in breast cancer-a metaanalysis of randomized trials. Radiat Oncol 8:267

51. Sautter-Bihl ML, Sedlmayer F, Budach W, Dunst J, Feyer P, Fietkau R et al (2014) DEGRO practical guidelines: radiotherapy of breast cancer III-radiotherapy of the lymphatic pathways. Strahlenther Onkol 190:342-351

52. o A (2005) Effects of chemotherapy and hormonal therapy for early breast cancer on recurrence and 15-year survival: an overview of the randomised trials. Lancet 365:1687-717

53. Galecki J, Hicer-Grzenkowicz J, Grudzien-Kowalska M, Michalska T, Zalucki W (2006) Radiation-induced brachial plexopathy and hypofractionated regimens in adjuvant irradiation of patients with breast cancer - a review. Acta Oncol 45:280-284

54. Haviland JS, Owen JR, Dewar JA, Agrawal RK, Barrett J, BarrettLee PJ et al (2013) The UK Standardisation of Breast Radiotherapy (START) trials of radiotherapy hypofractionation for treatment of early breast cancer: 10-year follow-up results of two randomised controlled trials. Lancet Oncol 14:1086-1094

55. Friberg S, Ruden BI (2009) Hypofractionation in radiotherapy. An investigation of injured Swedish women, treated for cancer of the breast. Acta Oncol 48:822-831

56. Darby SC, Ewertz M, McGale P, Bennet AM, Blom-Goldman U, Bronnum D et al (2013) Risk of ischemic heart disease in women after radiotherapy for breast cancer. N Engl J Med 368:987-998

57. Nielsen MH, Berg M, Pedersen AN, Andersen K, Glavicic V, Jakobsen EH et al (2013) Delineation of target volumes and organs at risk in adjuvant radiotherapy of early breast cancer: national guidelines and contouring atlas by the Danish Breast Cancer Cooperative Group. Acta Oncol 52:703-710

58. Hortobagyi GN (1998) Treatment of breast cancer. N Engl J Med 339:974-984

59. Khan SA, Stewart AK, Morrow M (2002) Does aggressive local therapy improve survival in metastatic breast cancer? Surgery 132:620-626 (discussion 6-7)

60. Rapiti E, Verkooijen HM, Vlastos G, Fioretta G, Neyroud-Caspar I, Sappino AP et al (2006) Complete excision of primary breast tumor improves survival of patients with metastatic breast cancer at diagnosis. J Clin Oncol 24:2743-2749

61. Gnerlich J, Jeffe DB, Deshpande AD, Beers C, Zander C, Margenthaler JA (2007) Surgical removal of the primary tumor increases overall survival in patients with metastatic breast cancer: analysis of the 1988-2003 SEER data. Ann Surg Oncol 14:2187-2194

62. Carmichael AR, Anderson ED, Chetty U, Dixon JM (2003) Does local surgery have a role in the management of stage IV breast cancer? Eur J Surg Oncol 29:17-19

63. Blanchard DK, Shetty PB, Hilsenbeck SG, Elledge RM (2008) Association of surgery with improved survival in stage IV breast cancer patients. Ann Surg 247:732-738

64. Babiera GV, Rao R, Feng L, Meric-Bernstam F, Kuerer HM, Singletary SE et al (2006) Effect of primary tumor extirpation in breast cancer patients who present with stage IV disease and an intact primary tumor. Ann Surg Oncol 13:776-782 
65. Fields RC, Jeffe DB, Trinkaus K, Zhang Q, Arthur C, Aft R et al (2007) Surgical resection of the primary tumor is associated with increased long-term survival in patients with stage IV breast cancer after controlling for site of metastasis. Ann Surg Oncol $14: 3345-3351$

66. Le Scodan R, Stevens D, Brain E, Floiras JL, Cohen-Solal C, De La, Lande B et al (2009) Breast cancer with synchronous metastases: survival impact of exclusive locoregional radiotherapy. J Clin Oncol 27:1375-1381

67. Ruiterkamp J, Ernst MF (2011) The role of surgery in metastatic breast cancer. Eur J Cancer 47:S6-22
68. Badwe RPV, Hawaldar R et al (2013) Surgery and radiotherapy may not benefit metastatic breast cancer patients treated with chemotherapy. SABCS Abstract S2-02

69. Soran AOV, Ozbas S, Karanlik H, Muslumanoglu M, Igci A, Canturk Z, Utkan Z, Ozaslan C, Evrensel T (2013) Local Surgery for Metastatic Breast Cancer. San Antonio Breast Cancer Symposium Abstract S2-03 San Antonio: US

70. Perez CA, Fu A, Onishko H, Hallahan DE, Geng L (2009) Radiation induces an antitumour immune response to mouse melanoma. Int J Radiat Biol 85:1126-1136 